\title{
SAMase of bacteriophage T3 inactivates E. coli's methionine Sadenosyltransferase by forming hetero-polymers
}

\author{
Hadas Simon-Baram¹, Daniel Kleiner ${ }^{1}$, Fannia Shmulevich¹, Raz Zarivach ${ }^{1,2}$, Ran Zalk ${ }^{2}$, Huayuan Tang ${ }^{3}$, Feng \\ Ding $^{3}$, Shimon Bershtein ${ }^{1}$
}

\begin{abstract}
${ }^{I}$ Department of Life Sciences, Ben-Gurion University of the Negev, Beer-Sheva, Israel; ${ }^{2}$ Macromolecular Crystallography and CryoEM Research Center, The National Institute for Biotechnology in the Negev, Ben-Gurion University of the Negev, Beer-Sheva, Israel; ${ }^{3}$ Department of Physics and Astronomy, Clemson University, Clemson, South Carolina, USA, simonha@post.bgu.ac.il
\end{abstract}

S-adenosylmethionine lyase (SAMase) of bacteriophage T3 degrades the intracellular SAM pools of the host E. coli cells, thus inactivating a crucial metabolite involved in plethora of cellular functions, including DNA methylation [1]. SAMase is the first viral protein expressed upon infection and its activity prevents methylation of the T3 genome. Maintenance of the phage genome in a fully unmethylated state has a profound effect on the infection strategy - it allows T3 to shift from a lytic infection under normal growth conditions to a transient lysogenic infection under glucose starvation [2]. Using single-particle Cryo-EM and biochemical assays, we demonstrate that SAMase performs its function by not only degrading SAM, but also by interacting with and efficiently inhibiting the host's methionine S-adenosyltransferase (MAT) - the enzyme that produces SAM. Specifically, SAMase triggers open-ended headto-tail assembly of E. coli MAT into an unusual linear filamentous structure in which adjacent MAT tetramers are joined together by two SAMase dimers. Molecular dynamics simulations together with normal mode analyses suggest that the entrapment of MAT tetramers within filaments leads to an allosteric inhibition of MAT activity due to a shift to low-frequency high-amplitude active sitedeforming modes. The amplification of uncorrelated motions between active site residues weakens MAT's ability to withhold substrates, explaining the observed loss of function. We propose that the dual function of SAMase as an enzyme that degrades SAM and as an inhibitor of MAT activity has emerged to achieve an efficient depletion of the intracellular SAM pools.

IMPORTANCE: Self-assembly of enzymes into filamentous structures in response to specific metabolic cues has recently emerged as a widespread strategy of metabolic regulation. In many instances filamentation of metabolic enzymes occurs in response to starvation and leads to functional inactivation. Here, we report that bacteriophage T3 modulates the metabolism of the host $E$. coli cells by recruiting a similar strategy - silencing a central metabolic enzyme by subjecting it to phage-mediated polymerization. This observation points to an intriguing possibility that virus-induced polymerization of the host metabolic enzymes might be a common mechanism implemented by viruses to metabolically reprogram and subdue infected cells.

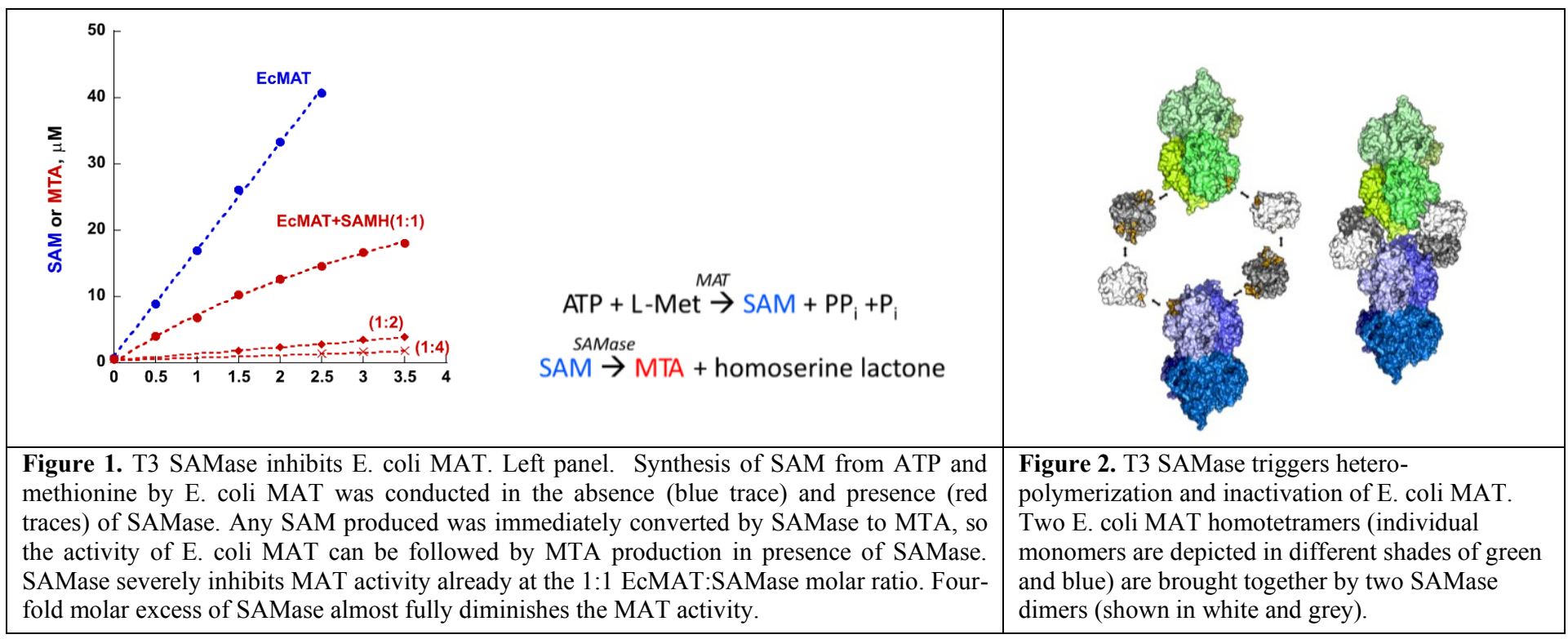

[1] Gefter, M., Hausmann, R., Gold, M. \& Hurwitz, J. J. Biol. Chem. 241, 1995-2006 (1966).

[2] Krueger, D. H., Presber, W., Hansen, S. \& Rosenthal, H. A.. J. Virol. 16, 453-455 (1975).

Keywords: enzyme filamentation, metabolic regulation, virus-host interaction, Cryo-EM, molecular dynamics (MD) simulations Acta Cryst. (2021), A77, C870 